\title{
EDITORIAL
}

\section{Auto-CPAP: an effective and low-cost procedure in the management of OSAS?}

\author{
P. Lévy, J-L. Pepin
}

Nasal continuous positive airway pressure (CPAP) is generally considered the first-line therapy in obstructive sleep apnoea (OSA) patients because it is effective at both correcting respiratory events and related arousals, and also improving morbidity [1]. Obstructive apnoeas and hypopnoeas can virtually always be eliminated by some level of CPAP $<20 \mathrm{cmH}_{2} \mathrm{O}[1,2]$. Determination of the prescription pressure (e.g. the lowest pressure that achieves a therapeutic result during an entire night), or "CPAP titration", has traditionally been conducted by a technician in the sleep laboratory during full polysomnography $[2,3]$. Although this procedure was initially exclusively directed towards eliminating only frank apnoeas and hypopnoeas, it is now common for the sleep technician to titrate CPAP first to the abolition of apnoeas and hypopnoeas, then to the elimination of snoring, intermittent oxygen desaturations and microarousals on electroencephalogram (EEG) [3, 4]. Until recently, CPAP titration was always carried out in the sleep laboratory, but this is a costly and labour-intensive procedure. Many strategies have been attempted to reduce this expense, including prediction formulae [5, 6], split night studies [7], attended home titration, partner titration and home stepwise titration. An alternative is to automate the titration process and determine a single pressure suitable for subsequent at-home fixed pressure treatment. The validity of such a strategy in correcting sleep breathing disorders has been demonstrated when compared with manual titration $[8,9]$. However, whether this permits a substantial reduction in or abolition of laboratory-based tests needed to titrate CPAP in OSA patients remains unknown.

In this issue of the Journal, BERKANI et al. [10] have examined the feasibility and economic impact on OSA management of an automated titration based on snoring detection. Although an adequate detection of the respiratory abnormalities on which the algorithm of the titrating device is based is the first condition of success, we will not comment further on this aspect as our group was involved in the development of the device used in the present report. However, several methodological points should be noted. An auto-adjusted CPAP device can be used either for titration only in order to choose the single therapeutic pressure that will be used on a long-term basis, or for adjusting permanently to the patient's changing needs during the night. Ideally a single device should be able to perform both [3], but in the present report, only the titration function was

Sleep and Respiration Unit, University Hospital of Grenoble, Grenoble France.

Correspondence: P. Lévy, EFCR, Hôpital A. Michallon, CHU Grenoble, 38043 Grenoble cedex, France. Fax: 33476765617. tested [10]. The authors have set several conditions to prevent any excess of sensitivity of the CPAP machine. In order to avoid any inappropriate increase in pressure: 1) the pressure was set at a maximal value of $14 \mathrm{cmH}_{2} \mathrm{O}$, or subsequently at 16 if previous threshold was attained; and 2 ) only snoring detection was used. This may be adequate for titration, but may be inadequate for long-term autoadjusted CPAP treatment. As a result of changes occurring in the upper airway structure and function (i.e. reduction of upper airway (UA) oedema and restoration of UA reflexes), snoring detection might become less effective in driving an auto-adjusted CPAP on a long-term basis. Moreover, although the authors carefully selected permanent snorers, the two subjects in whom the CPAP pressure adjustment was suboptimal had UA anomalies e.g. laryngectomy and uvulopalatopharyngoplasty (UPPP), respectively. It should be emphasized that UPPP is usually a contra-indication to auto-CPAP, whatever the device and indeed CPAP with fixed pressure is often less well-tolerated after such a surgical procedure [11].

There is little published uniformity to the criteria used by a technician to decide on the "optimal" pressure based on an attended study. Most protocols stress the abolition of apnoea which is relatively simple. The reference to hypopnoea is much more complex owing to the variability of detection between laboratories but also within a laboratory in this specific context of manual titration. Some laboratories also titrate CPAP to try to abolish arousals which may result from intermittent upper airway obstruction not producing detectable hypopnoea or desaturation. An additional approach that has been proposed is to use oesophageal pressure monitoring to titrate the level of CPAP to the pressure which minimizes intrathoracic pressure swings indicative of high resistance, thus correcting any elevated upper airway obstruction. A similar but noninvasive method is to use the inspiratory flow contour (flow limitation) to effect the CPAP titration [12]. Most, if not all the signals mentioned above are poorly standardized in terms of techniques of measurement and reference values. This is particularly the case for snoring measurement during CPAP which would be needed to provide comparable results between laboratories. Variability in hypopnoea detection has already been mentioned and is probably critical during an "on-line" procedure such as manual titration. Arousal detection is an even more complex issue as both the criteria used [13] and the normal values [14] remain questionable. Oesophageal pressure values may depend upon the technique of measurement but are also critically affected by sleep stages and body position. Thus, there have been virtually no normal values published until now [15]. Finally, 
inspiratory flow limitation may be recognized from flow contour but this is undoubtedly more easily and accurately done automatically by a specific algorithm than by visual inspection [8, 12]. On the other hand, the titration should not be performed only by increasing the pressure. There should be systematic attempts to lower the pressure through the night. This is required since the time spent at different pressures during the time study will strongly influence the choice of therapeutic pressure (i.e. 95 percentile of the pressure). It should also be borne in mind that some hysteresis exists in the pressure required to suppress respiratory events. This has been demonstrated with inspiratory flow limitation, which requires about $2 \mathrm{cmH}_{2} \mathrm{O}$ more to be eliminated during upwards titration of CPAP than the pressure at which it disappears during downwards titration [12].

Standardization of both the criteria and the procedure may favour auto-titration when compared with the manual procedure. Detection of respiratory events which require a change in CPAP pressure would be better addressed in an automated way. It is beyond the scope of the present editorial to recommend any strategy regarding the choice of signals or the design of algorithms. Several devices have already been designed using either pressure or flow monitoring to continuously adjust CPAP pressure. The parameters used to vary the pressure include apnoeas, hypopnoeas, snoring detection, inspiratory flow limitation assessment and respiratory impedance evaluation. From this point of view, there are two papers in the present issue of the Journal by ReEs et al. [16] and MAYER et al. [17] addressing the validity of simplified methods of diagnosis to assess arousals, apnoeas and hypopnoeas, respectively. Although these two papers are centred on the diagnostic side, the technology and the algorithms of many CPAP devices are already based on a similar strategy of detection. Whether one or more parameters are needed remains to be established. However, there are a number of factors which create significant difficulties for most, if not, all these devices. Buccal and nasal leaks when exceeding $0.5-1 \mathrm{~L} \cdot \mathrm{s}^{-1}$ (depending on the model) may greatly alter signal detection and consequently the adjustments of the device may be inadequate. In addition, rapid eye movement (REM) sleep, slow wave sleep and rapid alternation of wake/sleep periods are associated with physiological conditions which auto-adjusted CPAP devices have difficulty coping with: ventilatory instability, high stable UA resistance and repeated central events, respectively. There are technical solutions and algorithm designs that may partly solve these problems, but further improvement may be achieved by having a back-up system to control pressure changes which uses a different signal, should the original signal be uninterpretable for any reason.

There are two additional questions that need to be addressed regarding auto-titration: where should it be performed, and what is the cost-effectiveness of such a technique?

In the present report, the authors have attempted both to improve cost-effectiveness and to reduce the waiting list in their sleep laboratory [10]. It is not clear where the diagnostic night was performed, although it used an ambulatory device. However, the full polysomnography used to verify the efficacy of the fixed pressure provided by the auto-CPAP titration results was performed in a conven- tional hospital room. If one takes into account that a second titration would have been performed if the maximum pressure of $14 \mathrm{cmH}_{2} \mathrm{O}$ was attained, then the most significant finding of this study was a reduction in the number of polysomnographies, rather than an actual reduction in costs. In order to establish that there was a reduction, as the authors state, a careful comparison between the costs in the sleep laboratory and in the ward should be performed including the potential need for a second titration which could be required. In the paper by BeRKANI et al. [10] the number of subjects was small and none required a second titration. A larger study may have revealed extra costs in this respect. However, a reduced number of admissions to the sleep laboratory were undoubtedly obtained in the present study and this is, itself an important finding.

Furthermore, from these results we do not see any reason not to attempt auto-titration at home. There are recent reports proving the feasibility of such a procedure, although still published in abstract form $[18,19]$. However, several conditions should be fulfilled in order to be confident in the safety and efficacy of the procedure: 1) the auto-titrating device needs to be rigorously tested on a sufficiently large number of patients and proven to be working efficiently; 2) patients with significant mouth leaks e.g. those who have had UPPP should be excluded; 3) feedback from the patients and data recorded by the CPAP machine should be collected in the first days of treatment in order to detect rapidly any failure of the treatment; and 4) polysomnographic efficacy of the chosen pressure should be checked in the first weeks of treatment. If such a strategy is adopted, it might have several substantial advantages. It could generate a significant reduction in costs, and as previously mentioned, decrease the waiting list of the sleep laboratory which could be preferentially used for patients needing more sophisticated means of diagnosis and treatment. Moreover, the evaluation of treatment efficacy would be performed in a much more stable state, free of any sleep rebound effect and on a larger study period when compared to conventional titration. Finally, patients could be titrated at home over a period of several consecutive nights which would have the advantages of evaluating the reproducibility of the procedure and also of determining the CPAP pressure required to assess the needs in pressure for patients in their usual surroundings. Furthermore, there is also the option for patients requiring high or variable pressure to go on to the long-term use of an auto-titrating device. The proportion of patients requiring important changes in pressure through the night remains unknown. Whether this profile of pressure is stable over time is also uncertain. Therefore, this strategy would probably be able to select the most appropriate candidates for auto-CPAP on a long-term basis.

In conclusion, taking evidence from this latest report and from others already published or currently being conducted, it appears that auto-titration outside of the sleep laboratory is feasible and certainly cost-effective. It is important that the chosen auto-titrating device should have been validated and shown to be effective. Auto-titration at home over several nights is also a feasible and promising prospect. Whether or not subjects exhibiting significant variability in pressure-needs would benefit, in terms of side-effects and compliance, from long-term auto-adjusted continuous positive airway pressure remains to be studied. 


\section{References}

1. American Thoracic Society. Indications and standards for use of nasal continuous positive airway pressure (CPAP) in sleep apnea syndrome. Am J Respir Crit Care Med 1994; 150: 1738-1745.

2. Liistro G, Aubert G, Rodenstein D. Management of sleep apnea syndrome. Eur Respir J 1995; 8: 1751-1755.

3. Rapoport DM, Methods to stabilize the upper airway using positive pressure. Sleep 1996; 19: S123-S130.

4. Montserrat JM, Ballester E, Olivi H, et al. Time-course of stepwise CPAP titration: behaviour of respiratory and neurological variables. Am J Respir Crit Care Med 1995; 152: 1854-1859.

5. Hoffstein V, Mateika S. Predicting nasal continuous positive airway pressure. Am J Respir Crit Care Med 1994; 150: 486-488.

6. Sforza E, Krieger J, Bacon W, Petiau C, Zamagni M, Boudewyns A. Determinants of effective continuous positive airway pressure in obstructive sleep apnea: role of respiratory effort. Am J Respir Crit Care Med 1995; 151: 1852-1856.

7. Yamashiro Y, Kryger MH. CPAP titration for sleep apnea using a split-night protocol. Chest 1995; 107: 62-66.

8. Teschler H, Berthon-Jones M, Thompson A, Henkel A, Henry J, Kanietzko N. Automated continuous positive airway pressure titration for obstructive sleep apnea syndrome. Am J Respir Crit Care Med 1996; 154: 734-740.

9. Lloberes P, Ballester E, Montserrat J, et al. Comparison of manual and automatic CPAP titration in patients with sleep/hypopnea syndrome. Am J Respir Crit Care Med 1996; 154: 1755-1758.

10. Berkani M, Lofaso F, Chouaid C, et al. CPAP titration by an auto-CPAP device based on snoring detection: a clini- cal trial and economic considerations. Eur Respir J 1998; 12: 759-763.

11. Mortimore Il, Bradley PA, Murray JA, Douglas NJ. Uvulopalatopharyngoplasty may compromise nasal CPAP therapy in sleep apnea syndrome. Am J Respir Crit Care Med 1996; 154: 1759-1762.

12. Condos R, Norman RG, Krisnasam I, Peduzzi N, Goldering RM, Rapoport DM. Flow limitation as a noninvasive assessment of residual upper airway resistance during CPAP therapy of obstructive sleep apnea. Am J Respir Crit Care Med 1994; 150: 475-480.

13. ASDA. EEG arousals: scoring rules and examples. Sleep 1992; 15: 174-184.

14. Mathur R, Douglas NJ. Frequency of EEG arousals from nocturnal sleep in normals subjects. Sleep 1995; 18: 330 333.

15. Guilleminault C, Stoohs R, Clerk A, Cetel M, Maitros P. A cause of excessive daytime sleepiness: the upper airway resistance syndrome. Chest 1993; 104: 781-787.

16. Rees K, Wraith PK, Berthon-Jones M, Douglas NJ. Detection of apnoeas, hypopnoeas, and arousals by the Autoset in the sleep apnoea/hypopnoea syndrome. Eur Respir $J$ 1998; 12: 764-770.

17. Mayer P, Meurice JC, Philip-Joet F, et al. Simultaneous laboratory-based comparison of ResMed Autoset ${ }^{\mathrm{TM}}$ with polysomnography in the diagnosis of sleep apnoea/hypopnoea syndrome. Eur Respir J 1998; 12: 771-775.

18. Lantin M, Lacasse D, Remsbury S, et al. Home titration of nasal CPAP using an autotitrating CPAP machine. Sleep Res 1996; 25: 423.

19. Seriés F. Usefulness of an auto-CPAP home trial to determine the effective pressure level in the sleep apnea syndrome (SAS). Am J Respir Crit Care Med 1998; 157: A219. 